\title{
M. Decout, Qui a peur de l'imitation?
}

\section{Francesca Forcolin}

\section{(2) OpenEdition}

\section{Journals}

\section{Edizione digitale}

URL: http://journals.openedition.org/studifrancesi/10642

DOI: 10.4000/studifrancesi. 10642

ISSN: 2427-5856

\section{Editore}

Rosenberg \& Sellier

\section{Edizione cartacea}

Data di pubblicazione: 1 décembre 2017

Paginazione: 605-606

ISSN: 0039-2944

\section{Notizia bibliografica digitale}

Francesca Forcolin, «M. Decout, Qui a peur de l'imitation?», Studi Francesi [Online], 183 (LXI | III) | 2017,

online dal 01 février 2018, consultato il 13 février 2021. URL: http://journals.openedition.org/

studifrancesi/10642 ; DOI: https://doi.org/10.4000/studifrancesi.10642

\section{Questo documento è stato generato automaticamente il 13 février 2021.}

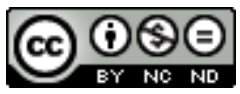

Studi Francesi è distribuita con Licenza Creative Commons Attribuzione - Non commerciale - Non opere derivate 4.0 Internazionale. 


\title{
M. Decout, Qui a peur de l'imitation?
}

\author{
Francesca Forcolin
}

\section{NOTIZIA}

MAXIME DECout, Qui a peur de l'imitation?, Paris, Les éditions de Minuit, 2017, 156 pp.

1 Maxime DECOUT, maitre de conférence all'Université Lille 3, fin dal titolo dell'«Introduction» (pp. 7-24) getta il lettore nel cuore dell'argomentazione, attraverso un artificio linguistico di volontaria e facile identificazione vista la notorietà del modello cui attinge: «Longtemps j'ai imité de bonne humeur». Oltre alla natura di tale artificio - un "pastiche", inteso come relazione con un testo altrui -, interessante è il fatto che esso porti già in sé gli snodi fondamentali che si andranno a delineare: "longtemps" (questione temporale), "je" (problematica identitaria), "de bonne humeur" (atteggiamento). Infatti, il rapporto con un testo di cui non si è firmatari, e più esattamente il sentimento di paura che l'imitazione scatena come il titolo di Decout suggerisce, è complesso e porta a una riflessione molto più ampia di quanto si possa credere: porta a soffermarsi sulla storia letteraria, sulle potenzialità della letteratura, sul concetto di originalità e di autenticità, sulla diposizione dello scrittore, nonché sulla nozione, essenziale, di identità. La storia letteraria, ci dice l'A., è segnata da un paradosso: dal rapporto ambivalente tra il fascino per l'imitazione e la sua paura, che muta evolvendo nel corso dei secoli portando a sempre nuove riflessioni sul ruolo dell'autore. Dagli antichi a oggi, da Orazio, La Fontaine, Pascal, Stendhal, Flaubert, Proust, Valéry, a Sarraute e Perec, i più grandi scrittori si sono dovuti confrontare con il demone dell'originalità e con il bisogno di autenticità, utilizzando tuttavia, nessuno escluso - più o meno consapevolmente, in maniera disinvolta o camuffata-le parole degli altri per scrivere le proprie. Tutti hanno immerso la propria penna nell'inchiostro altrui; nessuno può scrivere infatti a partire dal nulla, senza avere un bagaglio culturale (a volte parzialmente dimenticato, altre volte a portata di mano) cui attingere. Per gioco o per facilità, se non per svogliatezza, l'imitazione è dunque una pratica da sempre diffusa: lo facevano i grandi, da Virgilio a Shakespeare, a riprova che nella scrittura, come nelle arti in generale, l'importazione e l'appropriazione non negano la 
dimensione creatrice. Anzi, la ripresa e l'adattamento di parole, espressioni o frasi altrui generano a volte clins d'œil più o meno espliciti che accrescono le potenzialità della letteratura. Tuttavia, "de bonne humeur" o silenziosamente, il ricorso all'imitazione comporta sempre un approccio distanziato, quasi di inibizione o inferiorità rispetto al testo fonte, un pudore, un timore di accaparrarsi ciò che non spetta, di essere smascherati, o addirittura di perdere la propria identità. Lasciarsi influenzare o attingere a un testo altrui porta infatti a interrogarsi sul rapporto tra sé e il mondo, tra sé e l'altro, mettendo in campo sentimenti quali vergogna, colpevolezza, perdita di sé, diffidenza. Tale legame simbiotico tra sfera estetica ed esistenziale viene espresso da Decout attraverso un'analisi dettagliata, erudita e appassionata in due tempi. Il primo, «Malaises dans l'imitation» (première partie, pp. 25-82: «Chapitre I. Réprobations»; «Chapitre II. Justifications»; "Chapitre III. Vertiges identitaires»), riflette sulle contraddizioni e sull'ambiguità di tale pratica precisandone l'evoluzione. Alternativamente amata e ripudiata a seconda dei secoli, dei ritmi intellettuali, della Storia, delle società e delle mentalità, l'imitazione si vuole fenomeno naturale nella creazione letteraria. Riprendere e imitare sono infatti pratiche legittimate dal Rinascimento fino al XVII secolo, in quanto prova di grande erudizione da spartire con il lettore capace di cogliere le più sottili allusioni, ma anche simbolo di deferenza nei confronti degli antichi, depositari della tradizione a cui rimanere ancorati riducendo quanto più possibile il divario storico. Ma a partire dalla Rivoluzione francese si attua uno stravolgimento: l'estetica romantica, fermamente convinta dell'affermazione dell'io autoriale, fa trionfare una nuova pratica che vede nella cancellazione di ogni traccia di referenzialità esterna l'obiettivo a cui tendere. Il mimetismo scatena ormai timore e disgusto, come sancito lapidariamente da Hugo: «N'imitez rien ni personne». Bisogna attendere il xx secolo con la crisi del soggetto e la morte della parola ex nihilo perché l'imitazione faccia nuovamente il suo ingresso in scena e perché venga considerata come una delle possibili strade dell'espressione letteraria, naturalmente secondo modalità differenti rispetto al passato. L'imitazione diventa allora segno di una presa di coscienza e dimostrazione dell'impossibilità dell'atto originale, mettendo a nudo tutta la fragilità e l'inquietudine del tempo presente. Siamo proprietari delle nostre parole? Condannati alla ripetizione, siamo svuotati della nostra singolarità? Le parole si mescolano, si prestano, si scambiano volontariamente o meno, ma in ogni caso si apre la via al sospetto. Perché l'imitazione - minima o spudorata - porta a ragionare sull'appropriazione della parola altrui destando una vasta riflessione sulla proprietà intellettuale, che implica a sua volta i delicati concetti di plagio e diritto d'autore.

2 A partire da questi temi può prendere corpo la seconda parte, «De l'imitation comme défi à l'imitation» (deuxième partie, pp. 83-152), che comprende i capitoli «Imiter pour ne plus imiter», «Imiter pour corriger» e «Imiter pour repenser l'origine et l'originalité». Diviene ora possibile analizzare più da vicino l'ambiguità dell'imitazione, contesa tra il timore, l'angoscia e spesso l'euforia del gioco; una pratica di appropriazione tante volte esaltante e giubilatoria; ma anche una lotta per evitare o smussare i segni dell'altro, la sua intrusione, facendo spesso proprio dell'imitazione la migliore arma per paradossalmente sventare sé stessa. Di fronte all'incombenza di un maestro sovrano o alla difficoltà di raggiungere uno stile proprio senza scimmiottare quello altrui, ogni scrittore reagisce in maniera differente. Se alcuni utilizzano uno stile apparentemente piatto e neutro per bloccare l'ingresso ai segni distintivi dell'altro (Modiano, Ernaux), altri autori utilizzano il metodo scientifico, l'analisi testuale per mettere a distanza l'oggetto ammirato (Sartre nei confronti di Proust); altri ancora 
lottano inglobando fino all'estremo la voce dell'altro per bruciare le frontiere ed emanciparsi (Proust). I legami tra modello e copia sono pertanto decisamente complessi, ma lo sono in particolare, ci dice l'A., a partire da Flaubert, causa di una radicale modifica del nostro rapporto con il testo. Con Flaubert, autore eccelso a partire dal quale nulla è più come prima, si è sviluppata una particolare cesura intellettuale nella concezione del rapporto tra mimesi e scrittura, attuando una meditazione febbrile sulla nozione di originalità. Da qui, e in ultima istanza, Decout sonda la nozione di "origine", concetto evanescente e poroso: nel momento in cui le parole altrui scatenano la scrittura, si constata che l'autore non è più a monte della propria opera; parallelamente l'originalità non è più da intendersi opposta all'influenza ma in quanto dialogo profondo tra il sé e l'altro. Imitare, inoltre, significa anche partecipare alla diffusione del sapere, permettendo la circolazione del testo fonte che può a sua volta divenire modello per i posteri; è fondamento essenziale dello spazio letterario nonché forza distruttiva. Lo stile, pertanto - e la stimolante tesi di Decout mira a farcelo comprendere -, è un commercio identitario in cui l'io, tra timore e ammirazione, si rapporta con l'altro. La scrittura è un lavoro di elaborazione che passa attraverso la perdita di sé e l'accettazione dell'intrusione dell'altro, da cui, attraverso un movimento parallelo e opposto, necessariamente liberarsi. 